\title{
A case of successfully managed pregnancy in a patient with complex cyanotic congenital heart disease
}

\author{
JY Liu, WK Tan, EL Tan, JL Tan and LK Tan
}

\begin{abstract}
Medical advances have increased survival of patients with congenital heart disease. However, cardiac disease in pregnancy carries significant maternal and fetal risks, posing enormous challenges to obstetricians. Cyanotic congenital heart disease is associated with maternal complications such as arrhythmias, thromboembolic events and death. Fetal complications include small for gestational age, miscarriage and prematurity. Cyanotic congenital heart disease patients who continue their pregnancies require holistic multidisciplinary team care with early and coordinated planning for delivery. Management of such patients include early counseling regarding pregnancy-associated risks, close monitoring of their cardiac function and regular scanning for fetal assessment. Choice of anesthesia for these patients requires meticulous planning to achieve a favorable balance between systemic and pulmonary vascular resistance, ensuring minimal change in right-to-left shunting. We report a case of a successfully managed pregnancy in a patient with complex congenital heart disease and a single ventricle of left ventricle morphology.
\end{abstract}

\section{Keywords}

High-risk pregnancy, maternal-fetal medicine, cardiology, cardiac, cardiovascular

Date received: 24 May 2016; accepted: 17 October 2016

\section{Introduction}

Congenital heart disease (CHD) affects approximately $0.8 \%$ of newborns around the world. Over the past half-decade, medical advances with better diagnostic and surgical techniques have increased survival and decreased morbidity of patients with CHD, resulting in more than $85 \%$ surviving into adulthood. ${ }^{1,2}$ The incidence of non-syndromic major CHD in Singapore is about 2.6 per 1000 fetus, with a detection rate of $88.5 \%$ antenatally. ${ }^{3}$ With high detection rate and early treatment, the number of newborns with CHD reaching adulthood is expected to increase. Cardiac disease now affects an estimated $2 \%$ of pregnancies in developed countries. ${ }^{4} \mathrm{CHD}$, and complex cyanotic CHD in particular, carries significant risks for both mother and fetus. These cases pose enormous challenges to obstetricians and the multidisciplinary team looking after them. Univentricular heart or single ventricular heart is a rare congenital cardiac abnormality and to date, there are very few reported cases of successful pregnancies with this condition..$^{5-9}$ We report a case of a pregnancy in a 28 -year-old nulliparous with complex CHD and a single ventricle of left ventricle (LV) morphology.

\section{Presentation}

Our patient is a 28-year-old Chinese primigravidarum, who was referred at eight weeks of gestation to our hospital's high-risk pregnancy clinic. She was known to have complex CHD having presented at three weeks of life with congestive cardiac failure. Her diagnosis was dextrocardia, tricuspid atresia, hypoplastic right ventricle (RV) and transposition of great arteries with atrial septal defect and ventricular septal defect (VSD). At six weeks of life, she underwent pulmonary artery (PA) banding to decrease pulmonary blood flow. At the age of 19 years, she underwent bidirectional cavopulmonary connection (BCPC) with right pulmonary artery (RPA) patch-plasty. This required a balloon angioplasty for RPA segmental stenosis a year later. She also had a history of infective endocarditis and cerebrovascular accident from which she had made a full neurological recovery. A planned Fontan procedure could not be performed because she was found to have a large intracranial cortical atrio-venous malformation, and was judged to be at significant risk of intra-cranial bleeding during heart surgery.

At her booking antenatal visit, she reported exercise intolerance (NYHA Class II) and her baseline oxygen saturation was $82 \%$ on room air. On examination, she was normotensive and not tachycardic. There was clubbing of the fingers and central cyanosis. On auscultation, there was an ejection systolic murmur, and her lungs were clear with no crepitations. There was evidence of secondary erythrocytosis with a baseline hemoglobin $(\mathrm{Hb})$ level of $20-21 \mathrm{~g} / \mathrm{dl}$ and hematocrit (Hc) level of 55-60\%. An echocardiogram performed before her pregnancy showed dextrocardia with atrial and abdominal solitus. The BCPC was patent. The great arteries were transposed with the aorta arising from the RV and PA from the LV. There was evidence of previous PA banding, which had since narrowed down to $0.8 \mathrm{~cm}$, showing severe PA stenosis with peak gradient greater than $4 \mathrm{~m} / \mathrm{s}$. The peak PA pressure was $64 \mathrm{mmHg}$. The dominant LV was functioning well with an ejection fraction of $50-55 \%$. The RV was hypoplastic with tricuspid atresia. The VSD was large with bidirectional blood flow (Figure 1).

\section{Progress}

This was an unplanned pregnancy. Despite intensive counseling by her cardiologist regarding the significant risks of maternal and perinatal morbidity/mortality, she insisted on continuing with the pregnancy and would not consider a termination of pregnancy. She was referred to the multidisciplinary cardiology pregnancy clinic for pregnancy management from 12 weeks' gestation. She was closely followed up at the joint clinic subsequently.

Singapore General Hospital, Singapore, Singapore

\section{Corresponding author:}

JY Liu, Singapore General Hospital, 3I Third Hospital Avenue, \#03-03 Bowyer, Block C, Singapore 168753, Singapore.

Email: jiayi.liu@mohh.com.sg 
She was considered to be at increase risk of embolic events and was commenced on enoxaparin $40 \mathrm{mg}$ subcutaneous (sc) daily and low-dose aspirin $100 \mathrm{mg}$ daily for thromboprophylaxis.

At 16 weeks' gestation, she complained of increasing shortness of breath and difficulty with activities of daily living (NYHA class III). On admission, her oxygen saturation was $75 \%$ on room air, and there was significant cyanosis. Her $\mathrm{Hb}$ was $20.5 \mathrm{~g} / \mathrm{dl}$ and the $\mathrm{Hc}$ was $59.4 \%$. Echocardiography findings were stable from her baseline (Table 1). She was admitted and monitored in the high-dependency cardiac ward. A Doppler scan of her lower limbs to exclude venous thromboembolism was negative. The chest X-ray showed prominent pulmonary vasculature and no signs of consolidation.

Her deteriorating effort tolerance was attributed to increased shunting through the septal defects resulting in worsening cyanosis and reduced systemic oxygenation. The patient was again extensively counseled on her deteriorating cardiac condition and the risk of maternal morbidity and mortality, as well as the perinatal complications associated with fetal growth restriction and extreme prematurity. The option of termination of pregnancy was again refused. The multidisciplinary team comprising obstetricians, cardiologists, anesthetists, hematologists, neonatologists, and cardiothoracic surgeons met regularly and were updated of her clinical progress.

Fetal anomaly scans at $18+5$ weeks and $20+5$ weeks showed no obvious structural abnormalities or markers of aneuploidy. The fetus was thereafter assessed serially with growth and Doppler scans. At $25+4$ weeks, the fetus was appropriately grown with an estimated weight of $785 \mathrm{~g}$ with normal Doppler flow studies. Maternal echocardiogram was performed at two to four weekly intervals from her admission and did not show any significant changes from the baseline 2D echocardiogram.

During her hospital stay, her condition remained stable with minimal supplemental oxygen. Antenatal corticosteroids were administered at 24 weeks for acceleration of fetal lung maturity, and again at 26 weeks' gestation. At $26+3$ weeks, she had deteriorating dyspnea with worsening hypoxia. The multidisciplinary team made a decision to

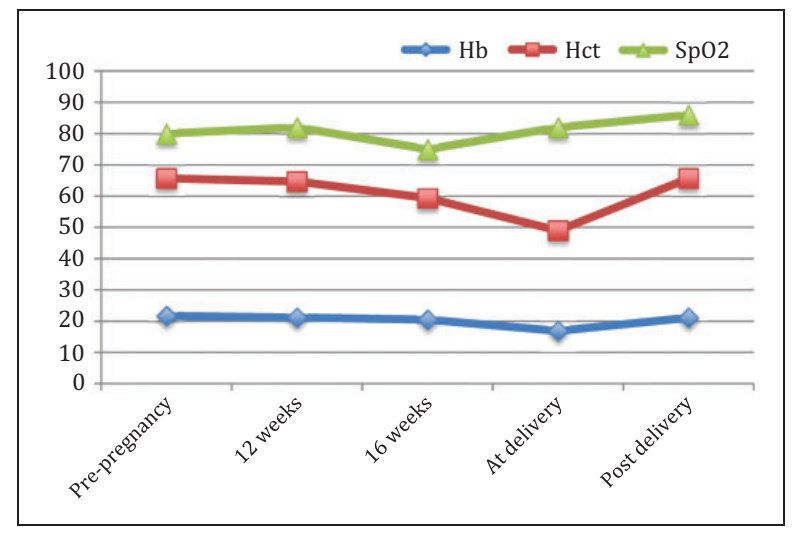

Figure I. Hemoglobin level, hematocrit and oxygen saturation trend of Mdm W. deliver. A classical caesarean section was planned in view of the extreme fetal prematurity and placenta praevia. She also consented to undergoing bilateral tubal sterilization at the time of delivery.

\section{Delivery}

General anaesthesia was chosen taking into consideration the issues of the surgically uncorrected right cortical brain arteriovenous malformation, scoliosis and lumbar prolapsed intervertebral discs. She was given $100 \mathrm{mcg}$ of fentanyl, $10 \mathrm{mg}$ of etomidate, $25 \mathrm{mg}$ of ketamine, and $50 \mathrm{mg}$ of rocuronium to facilitate rapid sequence induction. During intubation, the patient's blood pressure rose to $140 / 80 \mathrm{mmHg}$ but subsequently stabilized at $110 / 60 \mathrm{mmHg}$. She was given $100 \%$ supplemental oxygen prior to intubation and $75 \%$ oxygen thereafter. Her intra-operative oxygen saturation remained between $80 \%$ and $85 \%$. The cardiothoracic surgical team was placed on standby in the event of a need for extracorporeal membrane oxygen support.

She underwent an uneventful classical caesarean section and a baby boy, 870 grams, was delivered with APGAR scores of 4,4,6,9 at 1st, 5th, 10th and 20th minutes of life, respectively. Postoperatively, the patient was monitored in the cardiac intensive care unit. She received supplemental oxygen and thromboprophylaxis with enoxaparin $40 \mathrm{mg}$ sc daily and prophylactic antibiotics. She was also given three days of intravenous furosemide to prevent fluid overload. There was no requirement for inotropic support. She was well enough to be transferred to the general ward on the third post-operative day. Her oxygen saturation was stable at $84-86 \%$, and she was discharged well on 10 th postoperative day.

Three months after delivery, an echocardiography did not show any deterioration in LV ejection fraction and she had remained stable with no deterioration in her cardiac function or symptoms.

The newborn developed numerous complications related to extreme prematurity including hyaline membrane disease, pneumonia, neonatal sepsis, neonatal hypoglycaemia, and anemia of prematurity. The total duration of neonatal intensive care unit stay was 86 days and he was discharged home on day 122 of life. He has remained healthy subsequently, and has normal physical and neurological development during his pediatric follow-up. The child is currently seven months old.

\section{Discussion}

Single ventricle or univentricular heart is an uncommon form of CHD, accounting for about $3.2 \%$ of congenital cardiac abnormalities. ${ }^{10}$ It is defined by the presence of one dominant functioning ventricular compartment receiving the atria and giving rise to the pulmonary trunk, together with a rudimentary outlet chamber or a hypoplastic ventricle usually from tricuspid atresia (Figure 2). Such a condition is almost always associated with intracardiac mixing of deoxygenated systemic and oxygenated pulmonary venous return. Patients without natural pulmonary stenosis or surgical correction such as pulmonary banding or systemic pulmonary shunting are in danger of developing congestive heart failure early and pulmonary vascular disease, leading to pulmonary hypertension later on in life. Long-term survival for patients with univentricular hearts depends on achieving a balance between

Table I. The patient's increasing oxygen requirement during her admission.

\begin{tabular}{|c|c|c|c|c|c|c|c|}
\hline Gestation & 16 Weeks & 18 Weeks & 20 Weeks & 22 Weeks & 24 Weeks & 26 Weeks & Post-delivery \\
\hline $\mathrm{Min} \mathrm{SpO}_{2}(\%)$ & 75 & 80 & 82 & 80 & 83 & 82 & 84 \\
\hline $\mathrm{Max} \mathrm{SpO}_{2}(\%)$ & 95 & 98 & 92 & 89 & 91 & 87 & 86 \\
\hline Supplemental oxygen & Nasal prongs & Nasal prongs & Face mask & Face mask & Face mask & Non-rebreather & Non-rebreather \\
\hline Flow rate $(\mathrm{L} / \mathrm{min})$ & 3 & 3 & 4 & 4 & 4 & 6 & 6 \\
\hline
\end{tabular}


decreasing pulmonary arterial blood flow to relieve volume overload while simultaneously maintaining sufficient pulmonary blood flow for oxygenation of the blood. ${ }^{11}$

Pregnant women with cyanotic CHD are at increased risk for maternal and neonatal complications. Multiple studies have shown that maternal CHD is associated with a significant increase in adverse maternal outcomes, including cardiac failure, pulmonary hypertension, arrhythmias, pulmonary edema, cerebrovascular events, polycythemia, thromboembolic events, pregnancy induced hypertension, infective endocarditis, and death. ${ }^{2,12,13}$ Hemodynamic changes induced by pregnancy such as increased heart rate, stroke volume and cardiac output with parallel reduction of vascular resistance are particularly disadvantageous in women with single ventricular heart due to their significant dependence on venous return and limited capacity to increase the stroke volume. These physiological changes during pregnancy also exacerbate right-to-left shunting, leading to worsening maternal hypoxemia and cyanosis, which explained our patient's symptoms on admission at 16 weeks of gestation. ${ }^{14,15}$ Hence, the importance of close cardiac monitoring in such patients in anticipation of cardiac deterioration and decompensation.

Fetal complications from maternal CHD include miscarriage, small for gestational age (SGA), low birth weight, intrauterine demise, prematurity and its associated morbidities, as well as increased perinatal mortality. ${ }^{16-18}$ Chronic cyanosis further results in markedly increased risks of spontaneous abortion $(50 \%)$, premature delivery $(30-50 \%)$, and SGA. ${ }^{19}$ Fetal surveillance should begin early with a first trimester ultrasound to establish accurate dates and establishing baseline morphometry. Subsequent fetal growth and Doppler flow ultrasound studies are necessary to detect fetal growth restriction and placental insufficiency. As maternal CHD is also associated with a $16 \%$ increased risk of congenital fetal cardiac defects, ${ }^{17}$ detailed fetal anomaly scanning is recommended between 18 and 22 weeks. ${ }^{1,20,21}$

A study by Presbitero evaluating pregnancy outcomes of cyanotic CHD reported a favorable fetal outcome with pre-pregnancy arterial oxygen concentration at rest $>85 \%$ and hemoglobin concentration $<20 \mathrm{~g} / \mathrm{dL}$. In particular, fetal survival was $92 \%$ with maternal arterial saturations at $\geq 90 \%$ but was only $12 \%$ with arterial saturations $<85 \%$. ${ }^{16}$ Our patient's parameter was significantly below the threshold; her oxygen saturation was $75 \%$ on admission and her hemoglobin concentration was more than $20 \mathrm{~g} / \mathrm{dL}$. Predictors of poor pregnancy outcome and high maternal mobidity and mortality include the presence of pulmonary vascular disease, i.e pulmonary hypertension, Eisenmengers Syndrome, chronic maternal cyanosis, unrepaired or palliated maternal CHD, and poor systemic ventricular function. ${ }^{30-32}$ While there are classification systems such as CARPREG and ZAHARA to guide risk counseling, these are of limited utility in our patient. ZAHARA only had nine patients in a total cohort of 1302 women with complex cyanotic heart disease and tells little more than stating the obvious that she has a high-risk pregnancy. Moreover, the end points of ZAHARA are focused on events such as arrhythmias, whereas our patient was at high risk of much more significant outcomes including permanent impairment of cardiac function and even stroke. $^{22}$

Early planning for delivery is important in such high-risk pregnancies. While the timing of delivery always requires a balance between deteriorating maternal condition against fetal prematurity, in our patient, it was the former which was the deciding factor. The EPIcure study, a large prospective observational study evaluating outcomes of infants born before 26 weeks of gestation has demonstrated low survival rate $(39 \%)$; poor immediate and long-term outcomes in

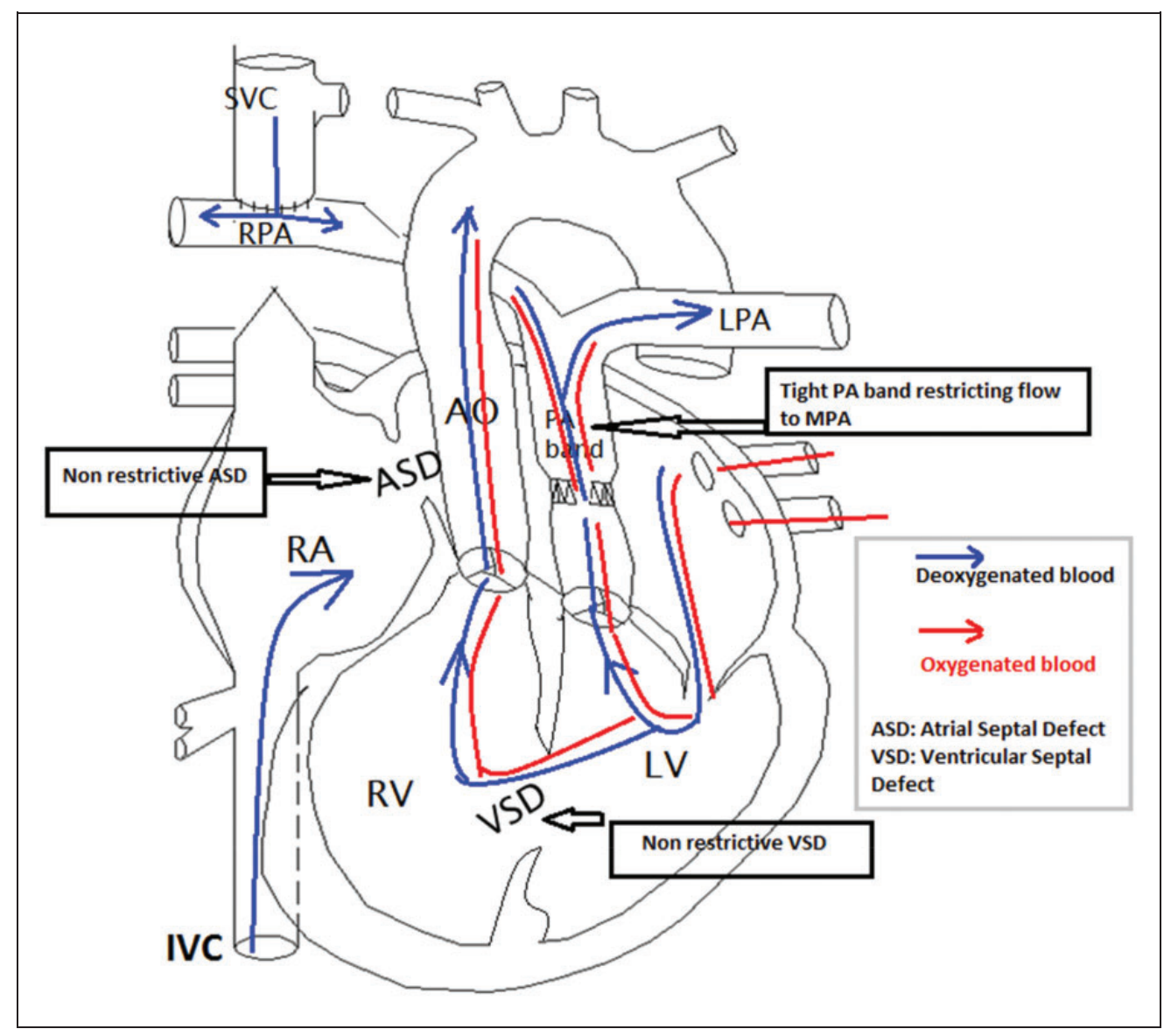

Figure 2. Diagrammatic demonstration of the patient's cardiac condition. 
these babies. ${ }^{23-25}$ Hence, delivery was initially aimed at 27 weeks after steroid completion. Intravenous magnesium sulfate for fetal neuroprotection was considered but held off in view of potential effect on myocardial function. ${ }^{26}$ The mode of delivery via caesarean section was uncontroversial in view of the severe fetal prematurity and the patient's cardiovascular constraints.

The anaesthetic issues were more complex. The goal of anaesthetic management in such patients should be to find a favorable balance between systemic and pulmonary vascular resistance to ensure minimal change in the amount of right-to-left shunt. ${ }^{27}$ In this case, general anaesthesia was preferred as it allowed greater control of the systemic vascular resistance. ${ }^{28}$ Furthermore, regional anaesthesia was deemed unsuitable for our patient in view of her multiple prolapsed intervertebral discs and history of cortical arteriovenous malformation. Routine antibiotics are not recommended for most mothers with heart disease; however, our patient was given three days of intravenous antibiotics peripartum in view of her history of infective endocarditis and her right-to-left shunt. ${ }^{29}$

In summary, pregnancy in patients with complex CHD is associated with significant maternal and fetal risks of morbidity and mortality. Successful outcome with a healthy, physically and neurologically intact live birth is uncommon. Patients who choose to continue their pregnancies require holistic care from a multidisciplinary team with early and coordinated planning for delivery. The integrated care from obstetricians, anaesthetists, cardiologist, pediatricians, hematologist, and midwives was unquestionably a key element to the successful management of our patient.

\section{Declaration of conflicting interests}

The author(s) declared no potential conflicts of interest with respect to the research, authorship, and/or publication of this article.

\section{Funding}

The author(s) received no financial support for the research, authorship, and/or publication of this article.

\section{Ethical approval}

Verbal and written consents have been obtained from the patient.

\section{Guarantor}

LKT

\section{Contributorship}

All authors contributed equally.

\section{References}

1. European Society of Gynecology, Association for European Paediatric Cardiology, German Society for Gender Medicine, Authors/Task Force Members, Regitz-Zagrosek V, Blomstrom Lundqvist C, et al. ESC guidelines on the management of cardiovascular diseases during pregnancy: the Task Force on the Management of Cardiovascular Diseases during Pregnancy of the European Society of Cardiology (ESC). Eur Heart J 2011; 32: 3147-3197.

2. Perloff JK. Historical perspective. In: Perloff JK and Child JS (eds) Congenital heart disease in adults. Philadelphia: WB Saunders, 1998, pp.3-8.

3. Chamaidi A and Gatzoulis MA. Heart disease and pregnancy. Hellenic J Cardiol 2006; 47: 275-291.

4. Dhanardhono $\mathrm{T}$ and Thia E. Incidence and outcome of prenatally diagnosed, chromosomally normal congenital heart defects in Singapore. Singapore Med J 2012; 53: 643-647.
5. Kinare SG, Sivaraman A and Deshpande J. Single ventricle (morphologic study of 21 cases). Indian Heart J 1989; 41: 301-306.

6. Theodoridis TD, Anagnostou E, Zepiridis L, et al. Successful pregnancy and caesarean section delivery in a patient with single ventricle and transposition of the great arteries. J Obstet Gynaecol 2005; 25: 69-70.

7. Amitha VK, Sahaja SK, Mallikarjun G, et al. Pregnancy outcome in a rare case of complex cyanotic congenital heart disease. Int $J$ Reprod Contracept Obstet Gynecol 2015; 4: 250-251.

8. Nilofer AR and Zaki SA. Successful pregnancy in a patient with univentricular heart and pulmonary stenosis. Heart Views 2012; 13: 71-73.

9. Wendling AL. Seller A and Peterson E. Case report of a parturient with cyanotic congenital heart disease palliated with a Glenn Procedure. J Clin Anesth 2012; 24: 586-589.

10. Rahimtoola SH, Ongley PA and Swan HJ. The haemodynamits of the common ventricle. Circulation 1966; 34: 14.

11. Steinberg EH and Dantzker DR. Single ventricle with severe pulmonary hypertension: natural survival into the third decade of life. Am Heart J 1993; 125: 1451-1453.

12. Optowsky A, Siddiqi O, D'Zouza B, et al. Maternal cardiovascular events during childbirth among women with congenital heart disease. Heart 2011; 98: 145-151.

13. Siu JM. Colman Cardiovascular problems and pregnancy: an approach to management. Cleve Clin J Med 2004; 71: 977-985.

14. Abbas AE, Lester SJ and Connolly H. Pregnancy and the cardiovascular system. Int J Cardiol 2005; 98: 179-189.

15. Cunningham FG, Leveno KJ, Bloom SL, et al. Williams obstetrics. In: Cunningham FG (ed) Cardiovascular disease. 23rd ed. USA: McGraw-Hill Companies, 2010, pp. 958-982.

16. Presbitero P, Somerville J, Stone S, et al. Pregnancy in cyanotic congenital heart disease. Outcome of mother and fetus. Circulation 1994; 89: 2673-2676.

17. Whittemore R, Hobbins J and Engle M. Pregnancy and its outcome in women with and without surgical treatment of congenital heart disease. Am J Cardiol 1982; 50: 6.

18. Gelson E, Curry R, Gatzoulis MA, et al. Effect of maternal heart disease on fetal growth. Obstet Gynecol 2011; 118(2 Pt 1): 364.

19. Regitz-Zagrosek V, Lundqvist CB, Borghi C, et al. Task Force on the management of cardiovascular diseases during pregnancy of the European Society of Cardiology: expert consensus document on management of cardiovascular diseases during pregnancy. Eur Heart $J$ 2003; 24: 761-781.

20. Warnes C, Williams R, Bashore T, et al. ACC/AHA 2008 guidelines for the management of adults with congenital heart disease: a report of the American College of Cardiology/American Heart Association Task Force on Practice Guidelines (writing committee to develop guidelines for the management of adults with congenital heart disease). J Am Coll Cardiol 2008; 52: e142-e263.

21. Rychik J, Ayres N, Cuneo B, et al. American Society of echocardiography guidelines and standards for performance of the fetal echocardiogram. J Am Soc Echocardiogr 2004; 17: 803-810.

22. Drenthen W, Boersma E, Balci A, et al. ZAHARA Investigators. Predictors of pregnancy complications in women with congenital heart disease. Eur Heart $J$ 2010; 31: 2124-2132.

23. Costeloe K, et al. The EPICure Study: outcomes to discharge from hospital for infants born at the threshold of viability. Pediatrics 2000; 106: 659-671.

24. Tamanna M, Enid MH, Jonathan M, et al. Neurological and developmental outcome in extremely preterm children born in England in 1995 and 2006: the EPICure studies. BMJ 2012; 345: e7961.

25. Johnson S, Hennessy E, Smith R, et al. Academic attainment and special educational needs in extremely preterm children at 11 years of age: the EPICure study. Arch Dis Child Fetal Neonatal Ed 2009; 94: F283-F289. 
26. Pryde PG and Mittendorf R. Contemporary usage of obstetric magnesium sulfate: indication, contraindication, and relevance of dose. Obstet Gynecol 2009; 114: 669.

27. Boyle RK. Anaesthesia in parturient with heart disease, a five-year review in an Australian tertiary hospital. Int J Obstet Anesth 2003; 12: $173-177$.

28. Buckland R and Pickett JA. Pregnancy and the univentricular heart: case report and literature review. Int $J$ Obstet Anesth 2000; 9: 55-63.

29. Nishimura RA, Otto CM, Bonow RO, et al. 2014 AHA/ACC guideline for the management of patients with valvular heart disease: a report of the American College of Cardiology/ American Heart Association Task Force on Practice Guidelines. J Am Coll Cardiol 2014; 63: e57-e185.

30. Avila WS, Grinberg M, Snitcowsky R, et al. Maternal and fetal outcome in pregnant women with Eisenmenger's syndrome. Eur Heart $J$ 1995; 16: 460-464.

31. Jones AM and Howitt G. Eisenmenger's syndrome in pregnancy. BMJ 1965; 1: 1627-1631.

32. Koos BJ. Clinical management of pregnant women with congenital heart disease. In: Pitkin RM (moderator) Pregnancy and congenital heart disease. Ann Intern Med 1990; 112: 445-454. 